\title{
DIVISION VII / WORKING GROUP GALACTIC CENTER
}

\author{
CHAIR \\ Joseph Lazio
}

\section{TRIENNIAL REPORT 2006 - 2009}

\section{Introduction}

The Working Group Galactic Center was created following a Business Meeting meeting of Division VII Galactic Systems, held with the concurrence of Division VIII Galaxies and the Universe, at the IAU XXVI General Assembly in Prague, 2006. The objective of the meeting was to highlight both recent progress on understanding the Galactic Center as well as to illustrate the way in which the center of the Milky Way Galaxy serves as a laboratory for understanding other galactic nuclei.

The scientific meeting itself featured talks across the wavelength range, from radio to $\gamma$-ray, on two general topics. The first topic was the Galactic Center itself, particularly with respect to probing Sgr A*, the central supermassive black hole. Among the papers presented were a description of the possibility of imaging in the radio the shadow of Sgr A* against its accretion disk, the theory and observations of IR and high-energy flares from Sgr A*, and the Galactic Center at very-high-energy (VHE) $\gamma$-rays. The second topic was the environment of the Galactic Center and its relation to other galactic nuclei. Among the papers presented were theoretical considerations on star formation in the strong gravitational potential of the Galactic Center, censuses of the stars in the inner parsecs, and how the Milky Way Galaxy's nucleus compares to that of other disk-dominated galaxies.

Additionally, part of the motivation for the formation of the WG was the series of successful Galactic Center Workshops (Falcke et al. 1999; Cotera et al. 2002; Schödel et al. 2006), focused workshops, that bring the Galactic Center research community together to discuss recent progress as well as plan for future observations.

\section{Developments within the past triennium}

Primary activity of the WG is to publish GCNews <www.aoc.nrao.edu/ gcnews/> the newsletter for Galactic Center research. GCNews has more than 300 subscribers and serves as a forum for keeping the community informed about recent events relating to Galactic center research. GCNews consists of two components, a newsletter and newsflashes. Newsflashes are distributed by email as new abstracts are received.

Newsletters are Web publications, with the following major sections. An Abstract section compiles abstracts received since the last publication of a newsletter. An Announcement section keeps the community informed about upcoming conferences, symposia, and workshops; as they become available, brief reports from previous conferences, symposia, and workshops are also presented. Finally, original feature articles headline the newsletter. The feature articles are designed to highlight recent results, explain aspects of Galactic center research in slightly more detail, or illustrate currently unresolved questions. In the past triennium, feature articles have included a re-evaluation of the extent to which 
non-thermal emission is present in the star forming region Sgr B (Lang, Palmer \& Goss 2008); a discussion of the mass function of black holes in the local Universe vis-á-vis the extent to which Sgr A* is 'typical' (Greene \& Ho 2007); and a discussion of stellar orbits in the central parsec and the implication for star formation in the Galactic center ( $\mathrm{Lu}$ et al. 2006).

A secondary focus for the WG has been planning for the next Galactic Center workshop. The previous three workshops have been held in either the United States or Germany, and there was broad agreement that it would be desirable to expand the sites considered for as hosts for GC'09. Given that the Galactic Center is overhead in the Southern Hemisphere, there was initial thought given to a Southern Hemisphere site. More recently, however, there has been an expression of interest from Asia, and it seems likely that the next Galactic Center workshop will be held there. Allowing for the prime observing season for the Galactic center being the (Northern Hemisphere) summer, the original target date was late in 2008. However, it was then decided that this might nonetheless be difficult to schedule with the Olympics. The current target date is in 2009, though careful attention is also being given to the dates of the IAU XXVII General Assembly in Rio de Janeiro, Brazil, so that GC'09 does not conflict with it.

\section{Closing remarks}

This triennium has seen a number of remarkable scientific discoveries and advances related to the Galactic center. High-energy observations have become routine and are producing an entirely new probe of the region. A number of hypervelocity stars, on escape trajectories from the Galaxy, have been identified and used to probe the conditions in the Galactic Center that gave rise to their extreme velocities. Infrared and $\mathrm{X}$-ray flares continue to provide strong constraints on the accretion environment around Sgr A*. And recent sub-millimeter very long baseline interferometric (VLBI) observations of Sgr A* continues to indicate the promise of future imaging of the accretion shadow of the supermassive black hole itself. With new instruments and techniques continuing to be developed, the next triennium looks quite exciting for continued understanding of the Galactic Center.

\section{Acknowledgements}

It is a pleasure to thank the other individuals responsible for the smooth running of GCNews during the past triennium, including Loránt O. Sjouwerman, Cornelia C. Lang, Rainer Schödel, Masaaki Sakano, Feng Yuan, and Robeson M. Herrnstein, as well as Angela S. Cotera and Heino D. Falcke, who started GCNews. Basic research in radio astronomy at the Naval Research Laboratory is supported by 6.1 Base funding.

\section{Joseph Lazio chair of the Working Group}

\section{References}

Cotera, A., Markoff, S., Geballe, T. R., \& Falcke, H. (eds.) 2002, The Central 300 Parsecs of the Milky Way, Proc. Galactic Center Workshop 2002, Astron. Nach., Suppl. 1

Falcke, H., Cotera, A., Duschl, W. J., Melia, F., \& Rieke, M. J. (eds.) 1999, The Central Parsecs of the Galaxy, Proc. Galactic Center Workshop 1998, ASP-CS 186

Greene, J. \& Ho, L. 2008, GCNews, 26

Lang, C., Palmer, P., \& Goss, M. 2008, GCNews, 27

Lu, J. R., Ghez, A. M., Hornstein, S. D., et al. 2006, GCNews, 25

Schödel, R., Bower, G. C., Muno, M. P., Nayakshin, S., \& Ott, T. (eds.) 2006, Proc. Galactic Center Workshop 2006, JPh-CS, 54 